\title{
Trends in local therapy application for early breast cancer patients in the Japanese Breast Cancer Society Breast Cancer Registry during 2004-2009
}

\author{
Shigehira Saji • Masahiro Hiraoka • \\ Yutaka Tokuda $\cdot$ Naohito Fukui $\cdot$ Tadashi Ikeda
}

Published online: 29 November 2011

(C) The Japanese Breast Cancer Society 2011

The Japanese Breast Cancer Society (JBCS) has been accumulating breast cancer patient data since 1975, reaching a total of 188,265 cases over 29 years. However, the recent increase of breast cancer incidence in Japan made it impossible for us to maintain the same data pool system for further analysis of trends in the disease. Therefore, the JBCS launched a new patient registration system in 2004 , including 147,192 cases during the period 2004-2009.

Herein, recent trends in local surgery, lymph node clearance, and radiation application are summarized. As shown in Table 1, annual numbers of registered patients have increased year on year. This is partly due to the increasing trend of breast cancer incidence in Japan, but mainly due to the increasing number of institutions/hospitals cooperating with the JBCS Breast Cancer Registry. The number of patients registered in the year 2004 was

\section{S. Saji}

Department of Target Therapy Oncology, Kyoto University

Graduate School of Medicine, Kyoto, Japan

M. Hiraoka $(\square)$

Department of Radiation Oncology and Image-applied Therapy,

Kyoto University Graduate School of Medicine, 54

Kawaharacho, Shogoin, Sakyo-ku, Kyoto 606-8507, Japan

e-mail: hiraok@kuhp.kyoto-u.ac.jp

\section{Y. Tokuda}

Department of Breast and Endocrine Surgery, Tokai University

School of Medicine, Isehara, Kanagawa, Japan

N. Fukui

Japan Clinical Research Support Unit, Tokyo, Japan

T. Ikeda

Department of Surgery, Teikyo University School of Medicine,

Tokyo, Japan
15,675 , compared with more than twice as many $(34,987$ patients) in 2009. Figure 1 shows the proportional trend in surgical procedures from 2004 to 2009. Our previous report showed that the number of cases with breast-conserving surgery (BCS) overtook those with modified or total mastectomy in 2003, and BCS cases continued to increase thereafter [1]. In our dataset, an increase of the BCS proportion could be noted until 2007. However, this increasing trend reached a plateau after 2007.

In the standard procedure of BCS, radiation therapy after surgery should be mandatory. In some cases, such as older patients or patients with minimum tumor, there might be a relative indication for BCS without radiation therapy. In the JBCS registry data, an increase of standard BCS with radiation therapy was observed from 2004 to 2008 (Fig. 2).

Furthermore, for patients with mastectomy and 4 or more positive nodes, radiation therapy of chest wall and local lymph node area should be considered. Figure 3 shows the increasing trend of radiation therapy from 2004 to $2008 / 2009$. Since it is unlikely that the number of patients with multiple nodal involvement has increased in recent years, the main reason for this trend may be awareness of standard application of radiation therapy in such patients across all Japanese institutions/hospitals.

Sentinel lymph node biopsy (SLNB) is the standard approach for the patient with clinical node-negative disease. Figure 4 indicates a continuous increase of patients who had only SLNB without further axillary lymph node dissection (ALND). Almost half of patients could avoid ALND in 2009, although over $80 \%$ of patients had to undergo this intensive surgery in 2004. This should be due to increased application of SLNB in a substantial fraction of Japanese institutions/hospitals. Also, as indicated by recent results of the American College of Surgeons 
Table 1 Type of surgery for breast cancer patients in 2004-2009

\begin{tabular}{|c|c|c|c|c|c|c|c|}
\hline & 2004 & 2005 & 2006 & 2007 & 2008 & 2009 & Total \\
\hline No operation & 16 & 28 & 32 & 36 & 40 & 47 & 199 \\
\hline Breast conserving & 7,848 & 10,920 & 12,256 & 14,765 & 18,120 & 20,785 & 84,694 \\
\hline Total mastectomy & 923 & 1,399 & 1,597 & 2,269 & 3,243 & 3,880 & 13,311 \\
\hline Modified & 6,609 & 7,500 & 6,946 & 7,477 & 8,599 & 9,797 & 46,928 \\
\hline Halsted radical & 92 & 80 & 76 & 67 & 86 & 172 & 573 \\
\hline Others & 182 & 207 & 265 & 249 & 225 & 296 & 1,424 \\
\hline Unknown & 5 & 10 & 5 & 19 & 14 & 10 & 63 \\
\hline Total & 15,675 & 20,144 & 21,177 & 24,882 & 30,327 & 34,987 & 147,192 \\
\hline No. of institutions & 288 & 322 & 318 & 377 & 477 & 535 & \\
\hline
\end{tabular}

From the data of the JBCS registration program

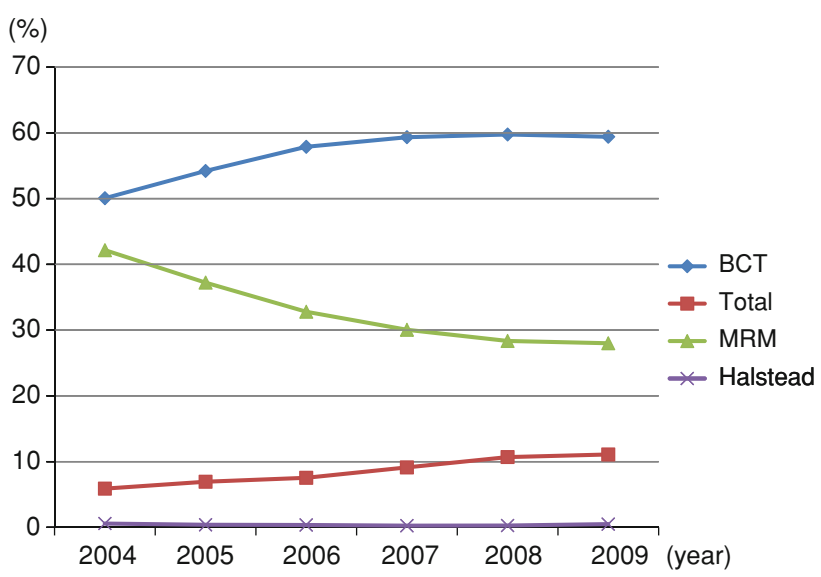

Fig. 1 Proportion of surgical procedures for breast cancer in Japan from 2004 to 2009 ( $n=147,192$ in total)

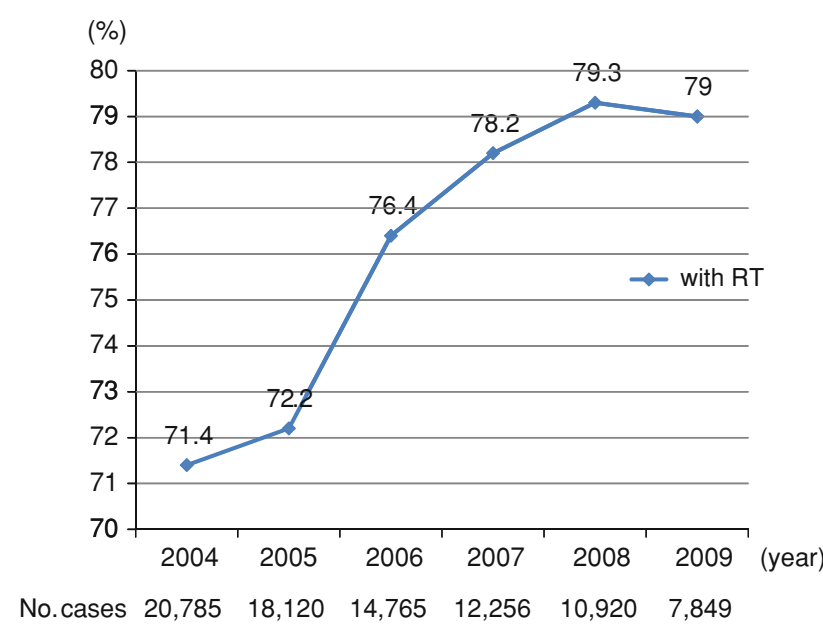

Fig. 2 Proportion of patients with radiation therapy (RT) after breastconserving surgery from 2004 to 2009 ( $n=84,694$ in total)

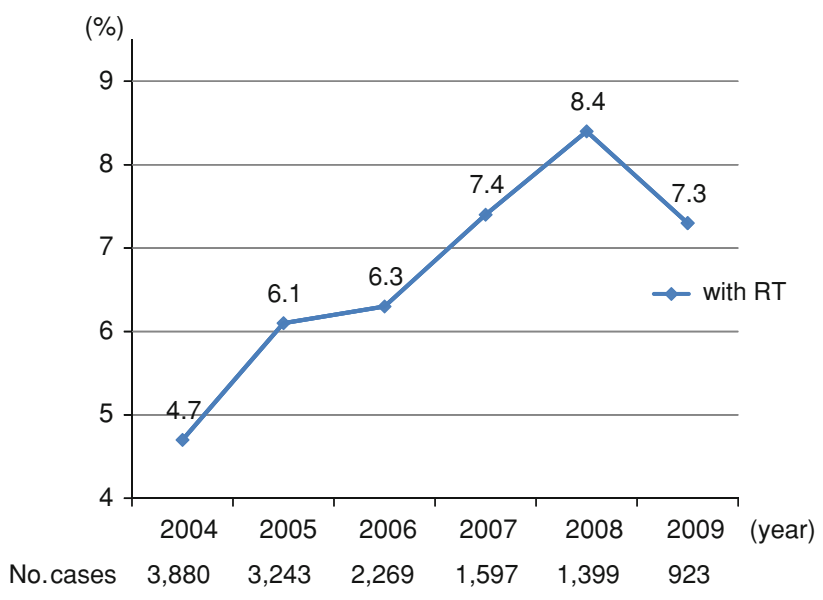

Fig. 3 Proportion of patients with radiation therapy (RT) after total mastectomy from 2004 to 2009 ( $n=13,311$ in total)

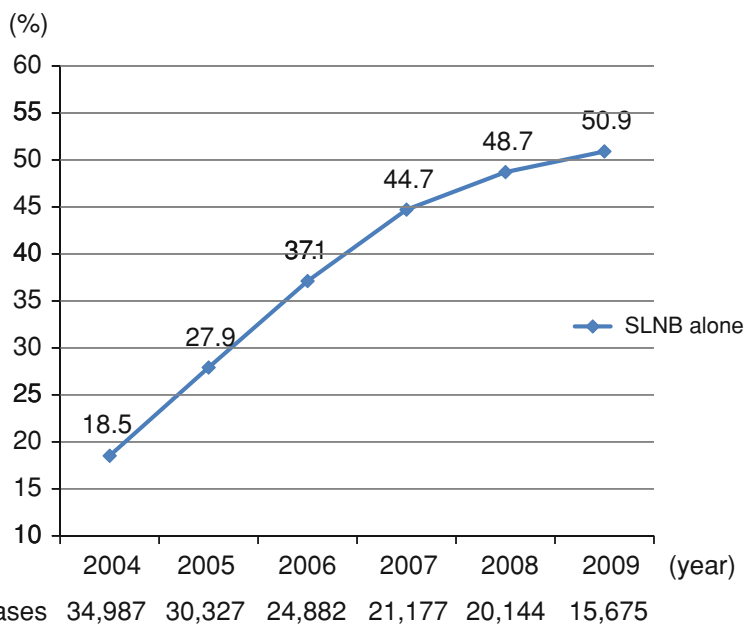

Fig. 4 Proportion of patients who had only sentinel lymph node biopsy without further axillary lymph node dissection (SLNB alone) from 2004 to 2009 ( $n=147,192$ in total) 
Oncology Group (ACOSOG) Z0011 trial [2], there was a demand to avoid unnecessary ALND for minimum lymph node involvement.

In the JBCS registry data from 2004 to 2009 , an increase of standard approach for surgical and radiation treatment could be found. Indication of BCS reached a plateau, and conversely, increased indication of mastectomy with or without breast reconstruction may occur in the next few years.

\section{References}

1. Sonoo H, Noguchi S, Academic Committee of the Japanese Breast Cancer Society. Results of questionnaire survey on breast cancer surgery in Japan 2004-2006. Breast Cancer. 2008;15(1):3-4.

2. Giuliano AE, Hunt KK, Ballman KV, Beitsch PD, Whiworth PW, Blumencranz PW, Leitch AM, Saha S, McCall LM, Morrow M. Axillary dissection vs no axillary dissection in women with invasive breast cancer and sentinel node metastasis: a randomized clinical trial. JAMA. 2011;305(6):569-75. 